\title{
CDDO-Me inhibits tumor growth and prevents recurrence of pancreatic ductal adenocarcinoma
}

\author{
XIAOHUA GAO $^{1}$, DORRAH DEEB ${ }^{1}$, YONGBO LIU ${ }^{1}$, PATRICIA LIU ${ }^{1}$, \\ YIGUAN ZHANG $^{2}$, JIAJIU SHAW ${ }^{2}$ and SUBHASH C. GAUTAM ${ }^{1}$
}

Departments of ${ }^{1}$ Surgery and ${ }^{2}$ Internal Medicine, Henry Ford Health System, Detroit, MI 48202, USA

Received August 10, 2015; Accepted October 8, 2015

DOI: 10.3892/ijo.2015.3212

\begin{abstract}
Methyl-2-cyano-3,12-dioxooleana-1,9(11)-dien28-oate (CDDO-Me) has shown potent antitumorigenic activity against a wide range of cancer cell lines in vitro and inhibited the growth of liver, lung and prostate cancer in vivo. In the present study, we examined the antitumor activity of CDDO-Me for pancreatic ductal adenocarcinoma (PDAC) cells with and without activating $K$-ras mutations. Treatment of K-ras mutant MiaPaCa-2 and K-ras normal BxPC-3 cells with CDDO-Me elicited strong antiproliferative and proapoptopic responses in both cell lines in culture. The inhibition of cell proliferation and induction of apoptosis was accompanied by the inhibition of antiapoptotic/prosurvival p-Akt, NF-KB and p-mTOR signaling proteins. For testing efficacy of CDDO-Me in vivo heterotopic and orthotopic xenografts were generated by implanting BxPC- 3 and MiaPaCa- 2 cells subcutaneously and in the pancreatic tail, respectively. Treatment with CDDO-Me significantly inhibited the growth of BxPC-3 xenografts and reduced the levels of p-Akt and p-mTOR in tumor tissue. In mice with orthotopic MiaPaCa-2 xenografts, treatment with CDDO-Me prolonged the survival of mice when administered following the surgical resection of tumors. The latter was attributed to the eradication of residual PDAC remaining after resection of tumors. These preclinical data demonstrate the potential of CDDO-Me for treating primary PDAC tumors and for preventing relapse/recurrence through the destruction of residual disease.
\end{abstract}

\section{Introduction}

Pancreatic ductal adenocarcinoma (PDAC) accounts for $>90 \%$ of all exocrine pancreatic cancers and is almost uniformly lethal with an estimated annual number of 45,220 new cases $\sim 38,460$ annual deaths and a 5-year survival rate of $<5 \%(1-3)$.

Correspondence to: Dr Subhash C. Gautam, Surgical Research - 4D, Henry Ford Health System, One Ford Place, Detroit, MI 48202, USA E-mail: sgautam1@hfhs.org

Key words: pancreatic ductal adenocarcinoma, CDDO-Me, apoptosis, Akt/mTOR/NF- $\mathrm{B}$ signaling, residual disease
Late initial diagnosis, aggressive metastatic behavior and resistance to chemo-radiotherapy render pancreatic cancer one of the most difficult to treat of all malignancies. Systemic chemotherapy with either gemcitabine or FOLFIRINOX (5-FU, irinotecan and oxaliplatin) is the current standard of care for advanced PDAC, providing short-term symptomatic improvement with minor impact on survival (4-6). Surgical resection is potentially curative; however, nearly $80 \%$ of the patients are diagnosed with locally advanced disease, precluding surgical intervention. Even in early stage PDAC patients in whom cancerous tissue is surgically resected (Whipple procedure), a majority of them relapse within two years after surgery. Thus, there is a tremendous need for novel therapeutics and treatment strategies for treating primary and residual PDAC for preventing relapse after pancreatic cancer surgery.

Aberration of apoptosis has been implicated in carcinogenesis and resistance to conventional anticancer therapies (7). Thus, promotion of apoptosis in cancer cells with novel agents could lead to tumor regression and improved prognosis. Triterpenes or triterpenoids are members of a large family of structurally related compounds known as cyclosqualenoids that are widely distributed in nature (8). Oleanolic acid is naturally occurring triterpenoid that has been used in traditional medicine as an anticancer and anti-inflammatory agent (9-11). In order to increase the anti-inflammatory and anticancer activity of oleanolic acid several synthetic analogues have been synthesized. Synthetic lealane triterpenoid 2-cyano-3,12dioxooleana-1,9(11)-dien-28-oic acid (CDDO) and its C-28 methyl ester derivative methyl-2-cyano-3,12-dioxooleana1,9(11)-dien-28-oate (CDDO-Me) and C-28 imidazole derivative 1-(2-cyano-3,12-dioxooleana-1,9-dien-28-oyl) imidazole (CDDO-Im) exhibit stronger anti-inflammatory activity than oleanolic acid $(12,13)$. CDDOs have also shown potent antiproliferative and proapoptotic activity in diverse types of tumor cell lines through the inhibition of MAPK (Erk1/2), $\mathrm{NF}-\kappa \mathrm{B}$ and PPAR $\gamma$ signaling pathways and they induce cytoprotective phase 2 response through Nrf2 signaling (14-20). In previous studies, we have demonstrated that the antiproliferative and apoptosis inducing activity of CDDO-Me in prostate cancer cell lines is mediated through the inhibition of antiapoptotic (prosurvival) Akt/NF- $\mathrm{B} / \mathrm{mTOR}$ signaling pathways (21). Although the antiproliferative activity of CDDOs in PDAC cell lines in vitro has been reported their therapeutic efficacy for PDAC in vivo has not been adequately studied. 
We have investigated the therapeutic efficacy of CDDO-Me against heterotopic and orthotopic tumor xenografts generated with human PDAC cell lines with a focus on preventing or delaying relapse/recurrence. Our data show therapeutic efficacy of CDDO-Me in treating primary tumor growth as well as in preventing/delaying recurrence of PDAC when administered after surgical removal of tumors.

\section{Materials and methods}

Reagents. CDDO-Me was obtained from the National Cancer Institute, Bethesda, MD, USA through the Rapid Access to Intervention Development Program. Antibodies against p-Akt $\left(\operatorname{ser}^{473}\right)$, p-mTOR $\left(\operatorname{Ser}^{2448}\right)$, PARP-1 and $\beta$-actin were purchased from Santa Cruz Biotechnology, Inc. (Santa Cruz, CA, USA). CellTiter 96 AQueous One Solution Proliferation assay system was from Promega (Madison, WI, USA) and Annexin V-FITC apoptosis detection kit II was obtained from BD Pharmingen (San Diego, CA, USA).

Cell culture. Human PDAC cell lines MiaPaCa-2 and BxPC-3 were obtained from the American Type Culture Collection (ATCC, Rockville, MD, USA). Cell lines were cultured at $37^{\circ} \mathrm{C}$ in a humidified atmosphere consisting of $5 \% \mathrm{CO}_{2}$ and 95\% air and maintained by subculturing cells twice a week.

Mice. Six-week-old female Scid/Ncr mice were purchased from the Charles River Laboratories, Inc., Frederic, MD, USA. Mice were maintained in a temperature-controlled room $\left(68-72^{\circ} \mathrm{F}\right)$ with a 12 -h light/dark cycle and provided semi-purified AIN-76A mouse chow and water ad libitum. Mice were acclimated for one week before starting the experiment. All animal treatments were according to the protocol approved by the Institutional Animal Care and Use Committee (IACUC).

Measurement of cell viability. Tumor cells $\left(1 \times 10^{4}\right)$ were seeded into each well of a 96-well plate in $100 \mu \mathrm{l}$ of tissue culture medium. After incubation at $37^{\circ} \mathrm{C}$ in a humidified atmosphere consisting of $5 \% \mathrm{CO}_{2}$ and $95 \%$ air for $24 \mathrm{~h}$, cells were treated with CDDO-Me at concentrations of 0.625-5 $\mu \mathrm{M}$ for $72 \mathrm{~h}$. Cell viability was then determined by the MTS assay using CellTiter $96^{\circledR}$ AQueous One Solution Proliferation assay system.

Measurement of apoptosis. Apoptosis was assessed by the binding of Annexin V-FITC to phosphotidylserine, which is externalized to the outer leaflet of the plasma membrane early during induction of apoptosis. Briefly, untreated cells and cells treated with CDDO-Me for $24 \mathrm{~h}$ were resuspended in the binding buffer provided in the Annexin V-FITC apoptosis detection kit and reacted with $5 \mu \mathrm{l}$ of Annexin V-FITC reagent and $5 \mu \mathrm{l}$ of propidium iodide (PI) for $30 \mathrm{~min}$ at room temperature in the dark. Stained cells were analyzed by flow cytometry using Accuri C6 flow cytometer (Accuri Cytometers Inc. Ann Arbor, MI, USA). The induction of apoptosis by CDDO-Me was confirmed by the cleavage of PARP-1 by western blotting.

Western blotting. Cell lysates were prepared using NP 40 cell lysis buffer. Lysates were clarified by centrifugation at
$14,000 \mathrm{x} \mathrm{g}$ for $10 \mathrm{~min}$ at $4^{\circ} \mathrm{C}$ and protein concentrations were determined. Samples $(50 \mu \mathrm{g})$ were boiled in an equal volume of sample buffer [ $20 \%$ glycerol, $4 \%$ SDS, $0.2 \%$ bromophenol blue, $125 \mathrm{mM}$ Tris- $\mathrm{HCl}$ ( $\mathrm{pH} 7.5)$, and $640 \mathrm{mM}$ 2-ME] and separated on pre-casted Tris-glycine polyacrylamide gels using the XCell Surelock ${ }^{\mathrm{TM}}$ Mini-Cell, in Tris-glycine SDS running buffer, all from Novex (Invitrogen, Carlsbad, CA, USA). Proteins resolved on the gels were transferred to PVDF membranes. Membranes were blocked with $5 \%$ milk in $10 \mathrm{mM}$ Tris- $\mathrm{HCl}(\mathrm{pH} 8.0), 150 \mathrm{mM} \mathrm{NaCl}$ with $0.05 \%$ Tween-20 (TPBS) and probed using specific antibodies against proteins of interest or $\beta$-actin (loading control) and HRP-conjugated secondary antibody. Immune complexes were visualized with enhanced chemiluminescence. Protein bands were imaged and band densities analyzed using the NIH/Scion image analysis software. The protein band densities were normalized to the corresponding $\beta$-actin band densities and percent change in signal strength was calculated.

Generation of tumor xenografts. Heterotopic PDAC xenografts were generated by implanting $1 \times 10^{6} \mathrm{BxPC}-3$ cells in $50 \mu \mathrm{l}$ of PBS subcutaneously in the right flank of 7-week-old Scid-Ncr female mice. Cells were allowed to form tumors for 7 days before initiating treatment with CDDO-Me. Orthotopic PDAC xenografts were generated by implanting MiaPaCa-2 cells stably expressing luciferase gene (MiaPaCa-2-Luc cells) in the pancreatic tail of Scid/Ncr mice. Briefly, mice were anesthetized with ketamine/xylazine (100/8 mg/kg i.p.) and a 2-cm long subcostal laparotomy was performed after clipping hair. Pancreas were exposed and $1 \times 10^{6}$ MiaPaCa-2-Luc cells in $20 \mu \mathrm{l}$ of PBS were injected in the pancreatic tail with a $30 \mathrm{G}$ needle. Proper deposition of cells was confirmed by bleb formation at the site of injection. The fascia and skin incisions were sutured following implantation of tumor cells.

Residual disease. To generate residual disease, $1 \times 10^{6} \mathrm{MiaPaCa}-$ 2-Luc cells in $20 \mu$ l of PBS were injected in the pancreatic tail as described above. Cells were first allowed to form tumors for 28 days and then mice were anesthetized with ketamine/ xylazine (100/8 $\mathrm{mg} / \mathrm{kg}$ i.p.) and partial pancreatectomy was performed to remove all visible tumor growth. After resection of tumors, incisions were sutured and mice were either treated or not with CDDO-Me.

Treatment with CDDO-Me. Two treatment paradigms were followed. To treat primary tumor growth, treatment was started on day 7 after implantation of BxPC-3 cells subcutaneously. To treat residual disease, treatment was started one day after resection of tumors on day 28 after implantation of MiaPaCa2-Luc cells in the pancreas. Mice in both treatment paradigms were administered CDDO-Me at a dose of $15 \mu \mathrm{mol} / \mathrm{kg}$ $(7.5 \mathrm{mg} / \mathrm{kg}), 5$ days a week in $0.1 \mathrm{ml}$ of vehicle consisting of cremophor-EL:DMSO:PBS (1:1:8) by oral gavage until day 40 (heterotopic xenografts) to treat primary tumor growth or day 100 (orthotopic xenografts) to treat residual disease. Control mice were treated with vehicle alone without CDDO-Me.

Bioluminecsent imaging (BLI). The growth progression of orthotopic PDAC xenografts generated by implanting MiaPaCa-2-Luc cells in the pancreas was measured 


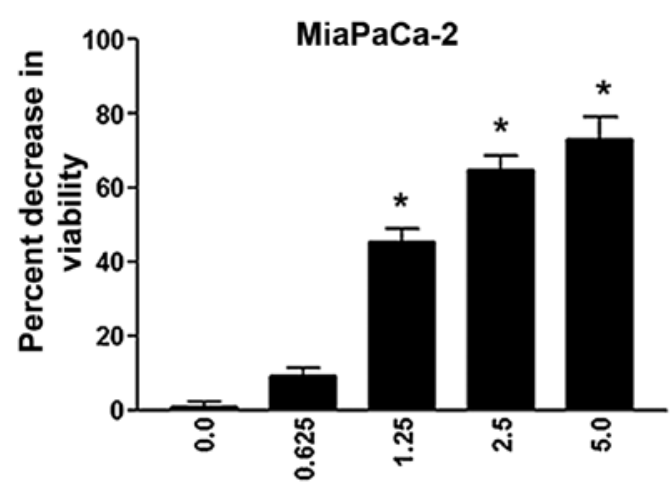

Concentration of CDDO-Me $(\mu \mathrm{M})$

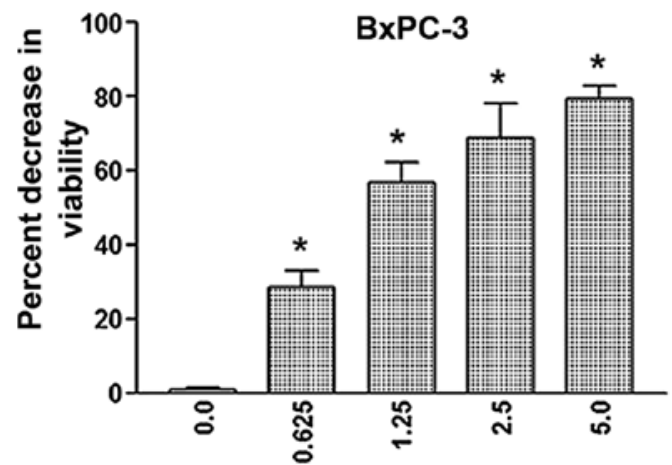

Concentration of CDDO-Me ( $\mu \mathrm{M})$

Figure 1. CDDO-Me inhibits the proliferation of PDAC cells. MiaPaCa- 2 or BxPC-3 cells $\left(1 \times 10^{4}\right)$ in $100 \mu 1$ of culture medium were seeded in each well of a 96-well microtiter plate and allowed to adhere for $24 \mathrm{~h}$. Cells were treated with CDDO-Me at concentrations ranging from 0 to $5 \mu \mathrm{M}$ for $72 \mathrm{~h}$ in triplicate. Cell viability was measured by MTS assay using CellTiter AQueous assay system from Promega. Data are presented as percent reduction in viability obtained in three independent experiments. ${ }^{*} \mathrm{p} \leq 0.05$ compared to control cells (no CDDO-Me).

non-invasively by BLI imaging using Kodak Multispectral Imaging system (Carestream Health, Woodbridge, CT, USA). Briefly, mice were anesthetized with isofluorane and anesthesia was maintained by inhalation of a mixture of isofluorane (2\%) and oxygen during the imaging procedure. First an X-ray image was taken and then BLI was obtained for $2 \mathrm{~min}, 10 \mathrm{~min}$ after i.p. injection of sodium luciferin $(150 \mathrm{mg} / \mathrm{kg})$. The X-ray and BLI images were overlaid and peak photon flux (photon/ $\mathrm{sec} / \mathrm{mm}^{2}$ ) from the tumors was plotted against time to obtain tumor growth curve.

Statistical analysis. Most data are presented as means \pm SD and outcomes for treated and untreated cells were compared by Student's t-test. Differences were considered significant at $\mathrm{p}<0.05$.

\section{Results}

CDDO-Me inhibits proliferation and induces apoptosis in PDAC cells. To test the effect of CDDO-Me on the proliferation of PDAC cells, $1 \times 10^{4} \mathrm{MiaPaCa}-2$ or $\mathrm{BxPC}-3$ cells were plated in 96-well microtiter plates for $24 \mathrm{~h}$ and then treated with CDDO-Me for $72 \mathrm{~h}$ at concentrations ranging from 0 to $5 \mu \mathrm{M}$. Viability of cultures was assessed by MTS assay and percent reduction in viability of cultures was determined. As shown in Fig. 1, significant decrease in the viability of cell cultures was observed in both cell lines treated with CDDO-Me at concentrations of 1.25-5 $\mu \mathrm{M}$ (MiaPaCa-2: 44-73\%; BxPC-3: 57-79\%).

To determine whether inhibition of proliferation of PDAC cells by CDDO-Me was associated with induction of apoptosis, binding of Annexin V-FITC to cells treated with CDDO-Me was measured. For this, MiaPaCa-2 or BxPC-3 cells were treated or not with CDDO-Me (0-5 $\mu \mathrm{M})$ for $24 \mathrm{~h}$ and analyzed for Annexin V-FITC binding by flow cytometry. As shown in Fig. 2A, the percentage of Annexin V-FITC binding MiaPaCa-2 cells increased from $<2 \%$ (untreated cells) to $17,24,37$ and $62 \%$ following treatment with CDDO-Me at $0.625,1.25,2.5$ and $5 \mu \mathrm{M}$, respectively. The percentage of Annexin V-FITC binding BxPC-3 cells was 1, 9, 16, 56 and $67 \%$ at $0,0.625,1.25,2.5$ and $5 \mu \mathrm{M}$ CDDO-Me.
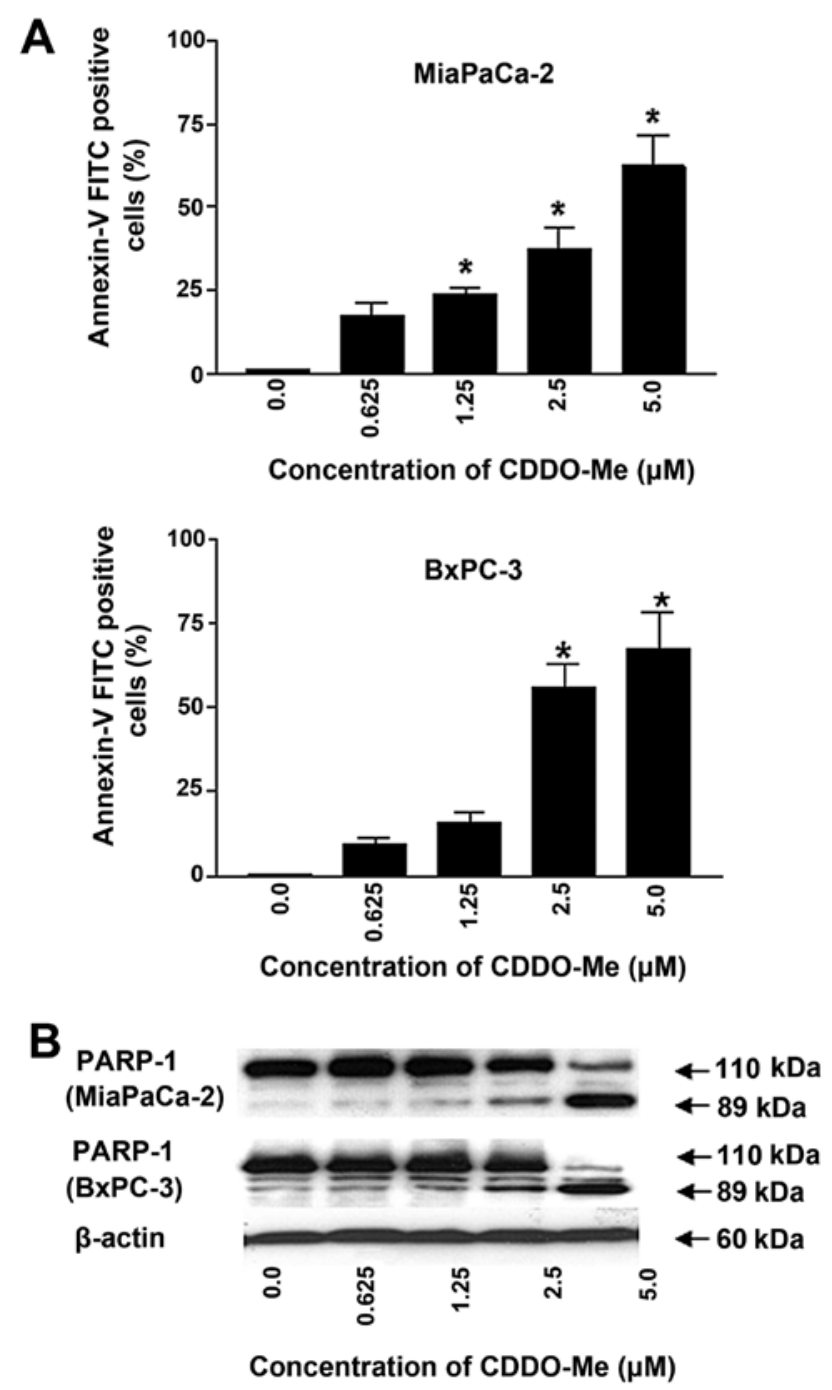

Figure 2. CDDO-Me induces apoptosis in PDAC cells. (A) Binding of Annexin V-FITC. MiaPaCa-2 cells and BxPC-3 cells were treated with CDDO-Me at concentrations of $0-5 \mu \mathrm{M}$ for $24 \mathrm{~h}$. Cells were then reacted with $5 \mu 1$ of Annexin V-FITC and $5 \mu 1$ PI for $30 \mathrm{~min}$ at room temperature in the dark. The percentage of Annexin V-FITC-positive tumor cells was determined by flow cytometry. (B) Cleavage of PARP-1. MiaPaCa- 2 cells and BxPC-3 cells were treated with CDDO-Me (0-5 $\mu \mathrm{M})$ for $24 \mathrm{~h}$. Cell lysates were analyzed for cleavage of PARP-1 by western blotting. Experiment was repeated twice. 

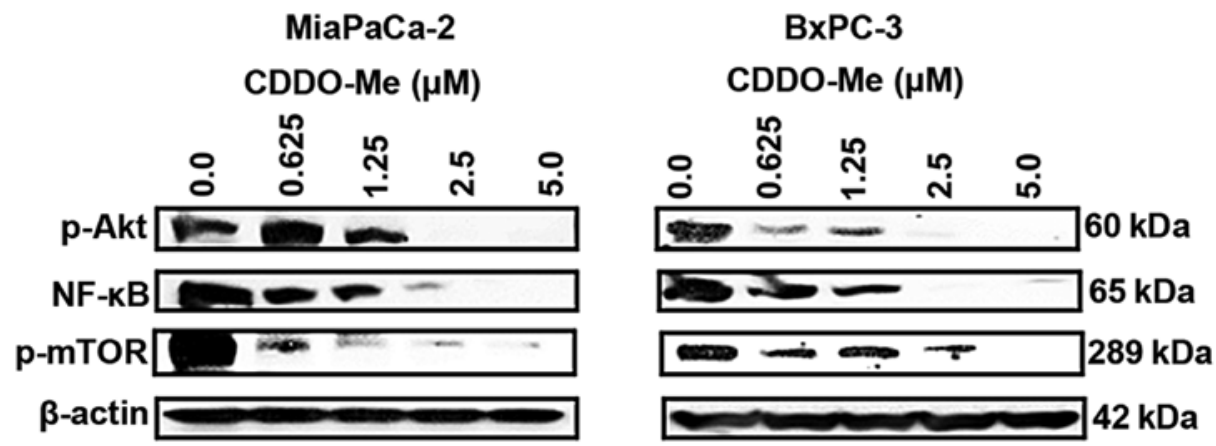

Figure 3. CDDO-Me downregulates prosurvival signaling proteins in PDAC cells. MiaPaCa-2 and BxPC-3 cells were treated with CDDO-Me (0-5.0 $\mu \mathrm{M})$ for $24 \mathrm{~h}$. Levels of p-Akt, NF-кB (p65), p-mTOR, and $\beta$-actin (loading control) were analyzed by western blotting. Experiment was repeated two times.

A

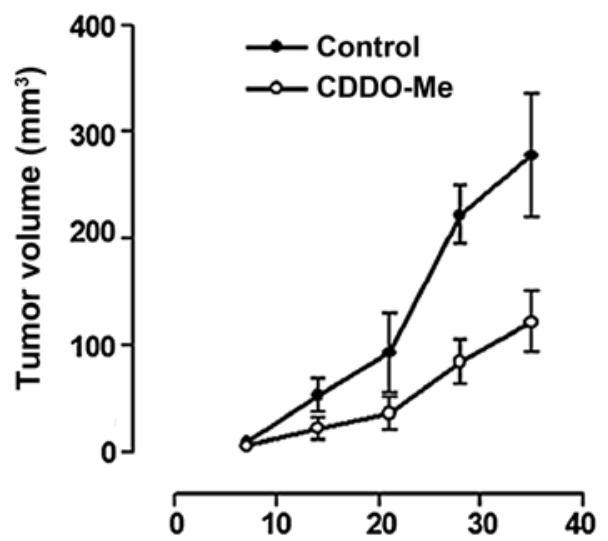

Days past tumor cell injection

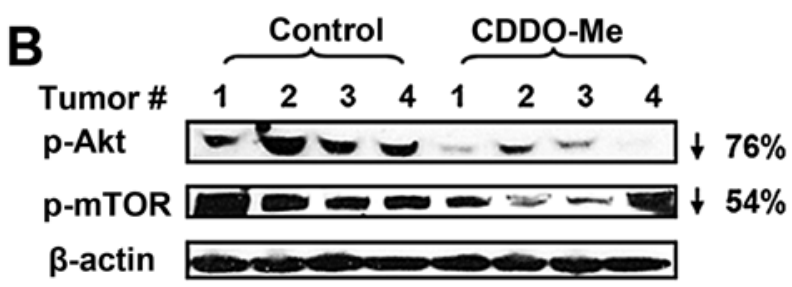

Figure 4. CDDO-Me inhibits growth of PDAC xenografts and expression of prosurvival signaling proteins in tumor tissue. (A) BxPC-3 cells $\left(1 \times 10^{6}\right)$ in $50 \mu 1$ of PBS were injected in the flank of seven-week-old female Scid/Ncr mice. Starting on day 7 after implantation of tumor cells mice were treated with CDDO-Me at a dose of $15 \mu \mathrm{mol} / \mathrm{kg}$ by oral gavage as described in Materials and methods. Control mice were treated with vehicle alone without CDDO-Me. Tumor dimensions (length and width) were measured with a caliper at weekly intervals. Data are plotted as tumor volumes vs days past tumor cell implantation \pm SD. (B) At termination of the experiment (day 40), tumor tissue from control and CDDO-Me treated mice was harvested and levels of p-Akt and p-mTOR were analyzed by western blotting.

The induction of apoptosis by CDDO-Me was confirmed by the cleavage of native PARP-1. As shown in Fig. 2B, treatment with CDDO-Me caused the emergence of $89 \mathrm{kDa}$ cleaved PARP-1 fragment in both cell lines. Together, increase in binding of Annexin V-FITC and cleavage of PARP-1 demonstrated induction of apoptosis by CDDO-Me in PDAC cells.

CDDO-Me inhibits $p$-Akt, NF- $\kappa B$ and $p$-mTOR signaling proteins in PDAC cells. Akt, NF-KB and mTOR are anti-apoptotic (prosurvival) signaling proteins that are constitutively active in a variety of human cancers, providing survival advantage to cancer cells. We investigated whether PDAC cells express constitutively active Akt (p-Akt) and mTOR (p-mTOR) and the effect CDDO-Me has on the expression of these signaling proteins. Cell lysates were prepared from MiaPaCa-2 and BxPC-3 cells treated or not with CDDO-Me $(0-5 \mu \mathrm{M})$ for $24 \mathrm{~h}$ and analyzed by western blotting for the levels of these proteins. Fig. 3 shows that both PDAC cell lines expressed p-Akt, NF-KB and p-mTOR and treatment with CDDO-Me significantly or completely inhibited their expression at concentrations of 1.25-5 $\mu \mathrm{M}$. These data demonstrated that induction of apoptosis by CDDO-Me is attributable at least in part to the inhibition of anti-apoptotic Akt, NF- $\mathrm{kB}$ and mTOR.

CDDO-Me inhibits the growth of BxPC-3 xenografts. Next we determined the tumor inhibitory activity of CDDO-Me in vivo against hetrotopic BxPC-3 xenografts. One week after injection of $1 \times 10^{6}$ BxPC-3 PDAC cells in the flanks of Scid-Ncr mice, mice were treated with CDDO-Me $(15 \mu \mathrm{mol} / \mathrm{kg})$ or vehicle only by oral gavage, 5 days/week for 5 weeks $(n=5)$ and experiment was terminated on day 40 . To measure the effect of CDDO-Me on progression of tumor growth, tumor dimentions (length and width) were measured by a caliper and tumor volume was calculated by the formula: (length $\mathrm{x}$ width $\left.{ }^{2}\right) / 2$. As shown in Fig. 4A, treatment with CDDO-Me significantly inhibited the growth of BxPC-3 xenografts compared to vehicle contol animals [tumor volume $\left(\mathrm{mm}^{3} \pm \mathrm{SD}\right)$ : control, $53 \pm 10,98 \pm 23,226 \pm 21$ and $284 \pm 39$; CDDO-Me, $22 \pm 6,37 \pm 10$, $79 \pm 16$ and $111 \pm 19$ on days $14,21,28$ and 35 after implantation of tumor cells]. Furthermore, treatment with CDDO-Me also reduced p-Akt and p-mTOR levels in the tumor tissue (76 and 54\% reduction, respectively) (Fig. 4B). These data demonstrated therapeutic efficacy of CDDO-Me against PDAC in vivo and provided evidence that activated Akt and mTOR are therapeutic targets of CDDO-Me in PDAC tumors.

Generation of orthotopic PDAC xenografts and efficacy of CDDO-Me for residual disease. To closely mimic physiological microenvironment of PDAC in patients for treatment with CDDO-Me we generated orthotopic xenografts by injecting MiaPaCa-2-Luc cells in the pancreatic tail in Scid/Ncr mice. The development and progression of orthotopic xenografts 

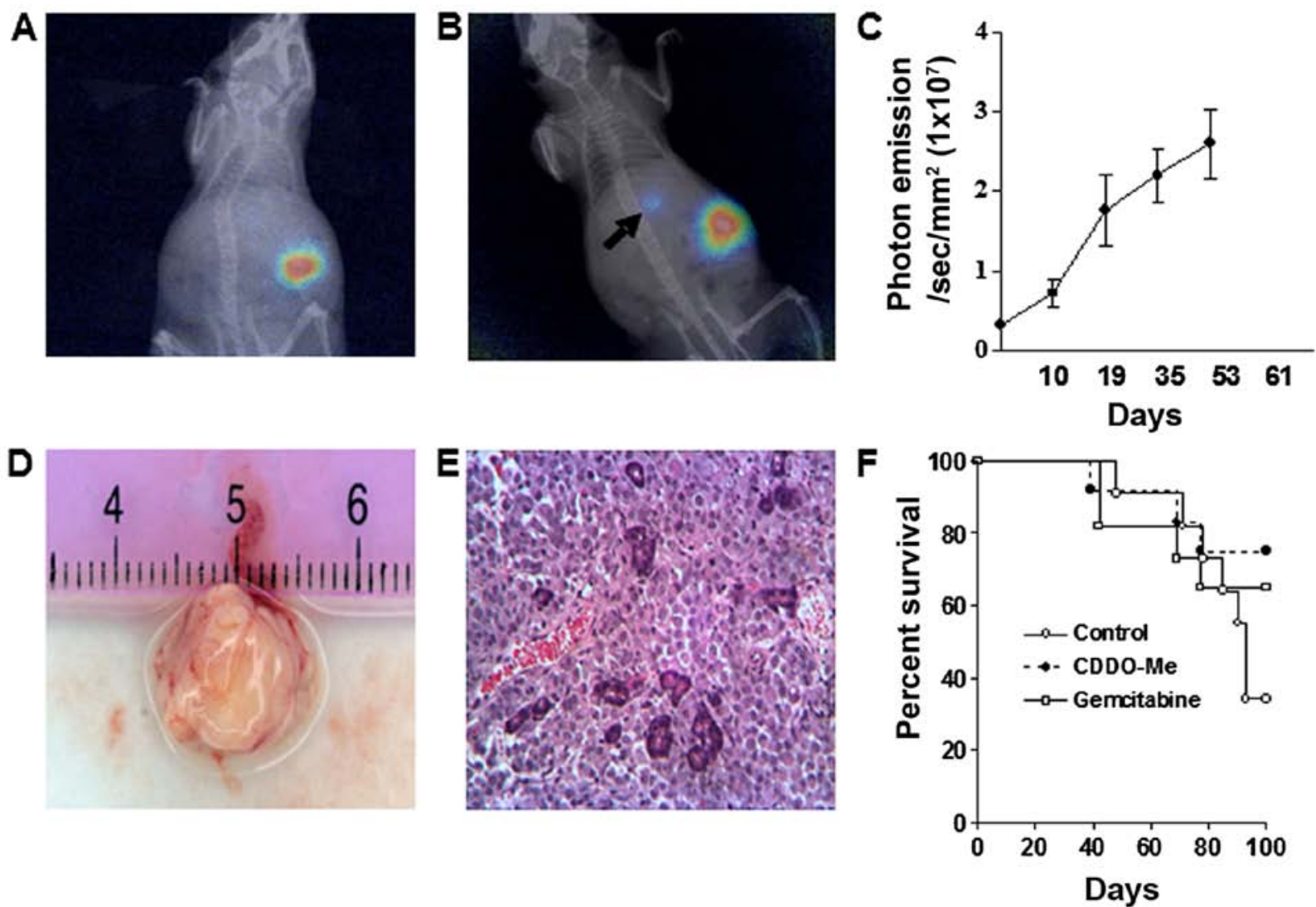

Figure 5. Bioluminescent imaging of orthotopic PDAC xenografts and efficacy of CDDO-Me in preventing recurrence. Female Scid/Ncr mice were injected with $1 \times 10^{6} \mathrm{MiaPaCa}-2$ cells stably expressing luciferase gene (MiaPaCa-2-Luc cells) in the pancreatic tail. The growth of xenografts was monitored by non-invasive bioluminescent imaging (A and B) and a growth curve was generated by plotting BLI signals from tumors (photon emission $/ \mathrm{sec} / \mathrm{mm}^{2}$ ) vs time (days) after implantation of MiaPaCa-2-Luc cells (C). Size and histology of a tumor harvested on day 35 is also shown (D and E). (F) To determine efficacy of CDDO-Me for preventing relapse, all visible tumor growth was resected on day 28 after implantation of MiaPaCa-2-Luc cells and mice were treated with CDDO-Me at a dose of $15 \mu \mathrm{mol} / \mathrm{kg}$ orally, 5 days a week until day 100. Control mice were treated with vehicle alone without CDDO-Me. In a separate group, mice were also treated with gemcitabine i.p., twice a week at a dose of $2 \mathrm{mg} / \mathrm{kg}$ to compare the activity of CDDO-Me to that of a standard agent for PDAC. Kaplan-Meier plots (F) represent survival data of vehicle only, gemcitabine or CDDO-Me treated animals.

was monitored non-invasively by bioluminescence imaging using Kodak Multispectral Imaging System (Carestream Health, Woodbridge, CT, USA). Fig. 5 shows BLI images of a xenograft on days 15 and 35 (Fig. 5A and B) and a metastatic lesion in the liver (Fig. 5B, arrow). The growth curve of xenografts was created by plotting photon flux emission (photons/ $\mathrm{sec} / \mathrm{mm}^{2}$ ) from tumors at various time points past tumor cell injection in the pancreas (Fig. 5C). The anatomical location of tumors was visually confirmed upon opening the abdominal cavity and tumors were harvested and identified as PDAC histologically (Fig. 5D and E).

We used the orthotopic xenogaft model to generate residual disease to investigate efficacy of CDDO-Me for preventing relapse (recurrence) after surgical removal of tumors. In this case, after injection of MiaPaCa-2-luc cells in the pancreatic tail, tumor cells were first allowed to form tumors. On day 28 , tumors were resected to remove all of the visible tumor growth in each animal. One day after removal of tumors, mice were treated with vehicle alone $(n=9)$ or CDDO-Me by oral gavage at a dose of $15 \mu \mathrm{mol} / \mathrm{kg}$ as described in Materials and methods $(n=12)$. Separately, mice were also treated with gemcitabine, the standard drug used for treating pancreatic cancer, at a dose of $2 \mathrm{mg} / \mathrm{kg}, 2$ times per week, i.p. $(\mathrm{n}=9)$. Experiment was terminated on day 100 and survival data were compared.
As shown in Fig. 5F, six of the nine mice in the vehicle control group died during this period (33\% survival; mean survival time $=81.6 \pm 22$ days). Five of the nine mice treated with gemcitabine survived during the same period $(56 \%$ survival; mean survival time $=81 \pm 25$ days, $p>0.05$ ). However, the group of mice treated with CDDO-Me showed the best survival rate, i.e., eight of the 12 mice treated with CDDO-Me revealed the presence of tumours in 2 of the 3 vehicle control, 2 of the 5 gemcitabine-treated and 1 of the 8 CDDO-Me-treated surviving mice on day 100.

\section{Discussion}

Pancreatic ductal adenocarcinoma is an intractable malignant disease with limited therapeutic options. Discovering effective novel agents with defined therapeutic targets might provide urgently needed therapeutics to treat this cancer. Natural products have long been recognized as a rich source of chemical diversity and compounds with a wide spectrum of anticancer activities. From vincristine to paclitaxel, the effective antitumor activity and novel mechanisms of action of many natural compounds and their synthetic derivatives have been described $(22,23)$. CDDO and CDDO-Me are synthetic triterpenoids derived from oleanolic acid with 
potent antiproliferative and pro-apoptotic activity. Earlier we demonstrated efficacy of CDDO and CDDO-Me in preventing/delaying the development and progression of prostate cancer in the transgenic TRAMP mouse model of prostate tumorigenesis $(24,25)$. Others have shown efficacy of CDDOs for breast, lung and colon cancers $(26,27)$. On the other hand, therapeutic efficacy of CDDOs for pancreatic cancer has not been adequately investigated. Studies described in this report were intended to determine therapeutic efficacy of CDDO-Me for pancreatic ductal adenocarcinoma. Since Kras is mutated in $>90 \%$ of human PDAC and mutation of Kras at codon G12 leads to constitutive activation of Kras oncoprotein and more aggressive PDAC phenotype (28), we investigated the anticancer activity of CDDO-Me in vitro and in vivo using two PDAC cell lines with and without activating $K$-ras mutations (MiaPaCa-2 and $\mathrm{BxPC}-3$, respectively). CDDO-Me strongly inhibited the proliferation of both cell lines in vitro independent of $K$-ras mutations. Since inhibition of cell proliferation induces apoptosis in cancer cells, we assessed induction of apoptosis by CDDO-Me in PDAC cells. Induction of apoptosis was measured by the binding of Annexin V-FITC to phosphotidylserine which is externalized to the outer leaflet of the plasma membrane early during induction of apoptosis. Treatment with CDDO-Me significantly increased the percentage of Annexin V-FITC binding cells in both cell lines. Induction of apoptosis was confirmed by the cleavage of PARP-1, a hallmark of cells undergoing apoptosis.

$\mathrm{PI} 3 \mathrm{~K} / \mathrm{Akt} / \mathrm{mTOR}$ and Akt/NF-kB are major antiapoptotic/ prosurvival signaling pathways that are constitutively active in most cancers including pancreatic cancer. Akt promotes cell growth and survival by inactivating downstream substrates such as Bad, procaspase-9 and forkhead transcription factors $(28,29)$. Antiapoptotic NF- $\mathrm{KB}$ and progrowth mTOR signaling pathways are downstream targets of activated PI3K/AKT. The $\mathrm{NF}-\kappa \mathrm{B}$ family of transcription factors controls the expression of genes involved in immune, inflammatory and oncogenic responses and play a critical role in resistance of cancer cells to anticancer therapies by protecting them from apoptosis (30). mTOR is a serine-threonine kinase, which controls cell growth, survival and ribogenesis (31). CDDO-Me inhibited the expression of $\mathrm{p}-\mathrm{AKT}, \mathrm{NF}-\kappa \mathrm{B}$ and $\mathrm{p}-\mathrm{mTOR}$ in MiaPaCa-2 and BxPC-3 cells. The inhibition of these anti-apoptotic and survival-promoting signaling proteins suggested that they are involved in mediating the antiproliferative and proapoptotic activity of CDDO-Me in PDAC cells.

The results of in vitro studies showing strong antiproliferative and proapoptotic activity of CDDO-Me for PDAC cells prompted us to evaluate its therapeutic efficacy for PDAC in vivo, since many times antitumor effects observed in cell lines in vitro do not pan out in vivo. We first examined CDDO-Me for therapeutic efficacy against heterotopic xenografts generated with BxPC-3 cells with normal $K$-ras expression. Treatment with CDDO-Me significantly inhibited the growth of heterotopic xenografts $(p<0.01)$. Western blot analysis of the tumor tissue extract showed that the inhibition of tumor growth was associated with the inhibition of antiapoptotic/prosurvival $\mathrm{p}-\mathrm{Akt}, \mathrm{NF}-\mathrm{kB}$ and $\mathrm{p}-\mathrm{mTOR}$ signaling proteins. These data also demonstrated that in vitro effects of CDDO-Me in PDAC cells does translate into therapeutic efficacy in vivo.
To further analyze therapeutic efficacy of CDDO-Me for PDAC, we tested it for preventing relapse/recurrence when administered after surgical resection of tumors. This was accomplished by creating a model of PDAC residual disease wherein orthotopic PDAC xenografts generated by implanting MiaPaCa-2-Luc cells in the pancreas were surgically removed after four weeks of tumor growth. Treatment with CDDO-Me in this adjuvant setting significantly prolonged survival of mice compared to untreated animals. In fact, the survival advantage with CDDO-Me was even better than that with gemcitabine, a standard drug for the treatment of patients with advanced PDAC. Thus, therapeutic outcomes in xenografts models generated with PDAC cells expressing either normal $K$-ras (BxPC-3) or activated $K$-ras (MiaPaCa-2) correlated with responses to $\mathrm{CDDO}-\mathrm{Me}$ in vitro. Although the exact mechanism of the antitumor activity of CDDO-Me in vivo remain to be investigated, our data suggest that downregulation of p-Akt, NF-кB and p-mTOR signaling proteins plays a role in inhibition of cell proliferation and induction of cellular apoptosis in tumor cells by CDDO-Me. Since treatment with gemcitabine or CDDO-Me alone improved survival when administered after removal of tumors suggests that combinatorial regimen of gemcitabine and CDDO-Me might be even more beneficial in preventing/delaying recurrence. This inference of course needs more rigorous analysis in these preclinical models of PDAC.

In conclusion, our investigations show that CDDO-Me exhibits therapeutic efficacy in xenograft models of PDAC (heterotopic and orthotopic) for inhibiting primary tumor growth and delaying/preventing relapse by destroying residual disease. Because of its ability to inhibit p-AKT, NF- $\kappa \mathrm{B}$ and p-mTOR in tumor tissue, we speculate that CDDO-Me inhibits tumor growth by inhibiting cell proliferation and promoting apoptosis through the inhibition of anti-apoptotic/prosurvival AKT/NF- $\mathrm{B} / \mathrm{mTOR}$ signaling pathways. Collectively, these findings unveiling antitumor potential of CDDO-Me for PDAC warrant further studies for development of CDDO-Me as an adjuvant to existing PDAC therapies to obtain more durable responses and improved outcomes.

\section{Acknowledgements}

This study was supported by NIH grant 1R01 CA130948 and a grant from Elsa U. Pardee Foundation. Authors thank Dr Ali S. Arbab for helping with BLI imaging.

\section{References}

1. Pancreatic Cancer-National Cancer Institute: U.S. National Institutes of Health. Cancer. Gov. http:www.cancer.gov/cancertopics/types/pancreatic. Accessed April 6, 2010.

2. Maitra A and Hruban RH: Pancreatic cancer. Annu Rev Pathol 3: 157-188, 2008.

3. Li D, Xie K, Wolff R and Abbruzzese JL: Pancreatic cancer. Lancet 363: 1049-1057, 2004.

4. Mulcahy MF, Wahl AO and Small W Jr: The current status of combined radiotherapy and chemotherapy for locally advanced or resected pancreas cancer. J Natl Compr Canc Netw 3: 637-642, 2005.

5. Pino SM, Xiong HQ, McConkey D and Abbruzzese JL: Novel therapies for pancreatic adenocarcinoma. Curr Oncol Rep 6: 199-206, 2004.

6. Vaccaro V, Sperduti I and Milella M: FOLFIRINOX versus gemcitabine for metastatic pancreatic cancer. N Engl J Med 365: 768-769, author reply 769, 2011. 
7. Kerr JF, Winterford CM and Harmon BV: Apoptosis. Its significance in cancer and cancer therapy. Cancer 73: 2013-2026, 1994.

8. Dzubak P, Hajduch M, Vydra D, Hustova A, Kvasnica M, Biedermann D, MarkovaL, Urban Mand Sarek J: Pharmacologica activities of natural triterpenoids and their therapeutic implications. Nat Prod Rep 23: 394-411, 2006.

9. Huang MT, Ho CT, Wang ZY, Ferraro T, Lou YR, Stauber K, Ma W, Georgiadis C, Laskin JD and Conney AH: Inhibition of skin tumorigenesis by rosemary and its constituents carnosol and ursolic acid. Cancer Res 54: 701-708, 1994.

10. Nishino H, Nishino A, Takayasu J, Hasegawa T, Iwashima A, Hirabayashi K, Iwata S and Shibata S: Inhibition of the tumorpromoting action of 12-O-tetradecanoylphorbol-13-acetate by some oleanane-type triterpenoid compounds. Cancer Res 48: 5210-5215, 1988

11. Ryu SY, Oak MH, Yoon SK, Cho DI, Yoo GS, Kim TS and Kim KM: Anti-allergic and anti-inflammatory triterpenes from the herb of Prunella vulgaris. Planta Med 66: 358-360, 2000.

12. Honda T, Rounds BV, Gribble GW, Suh N, Wang Y and Sporn MB: Design and synthesis of 2-cyano-3,12-dioxoolean1,9-dien-28-oic acid, a novel and highly active inhibitor of nitric oxide production in mouse macrophages. Bioorg Med Chem Let 8: 2711-2714, 1998.

13. Suh N, Honda T, Finlay HJ, Barchowsky A, Williams C, Benoit NE, Xie QW, Nathan C, Gribble GW and Sporn MB: Novel triterpenoids suppress inducible nitric oxide synthase (iNOS) and inducible cyclooxygenase (COX-2) in mouse macrophages. Cancer Res 58: 717-723, 1998

14. Ito Y, Pandey P, Sporn MB, Datta R, Kharbanda S and Kufe D The novel triterpenoid CDDO induces apoptosis and differentiation of human osteosarcoma cells by a caspase- 8 dependent mechanism. Mol Pharmacol 59: 1094-1099, 2001.

15. Konopleva M, Tsao T, Estrov Z, Lee RM, Wang RY, Jackson CE, McQueen T, Monaco G, Munsell M, Belmont J, et al: The synthetic triterpenoid 2-cyano-3,12-dioxooleana-1,9-dien-28-oic acid induces caspase-dependent and -independent apoptosis in acute myelogenous leukemia. Cancer Res 64: 7927-7935, 2004.

16. Gao X, Deeb D, Jiang H, Liu Y, Dulchavsky SA and Gautam SC: Synthetic triterpenoids inhibit growth and induce apoptosis in human glioblastoma and neuroblastoma cells through inhibition of prosurvival Akt, NF-kappaB and Notch1 signaling. J Neurooncol 84: 147-157, 2007.

17. Konopleva M, Contractor R, Kurinna SM, Chen W, Andreeff M and Ruvolo PP: The novel triterpenoid CDDO-Me suppresses MAPK pathways and promotes p38 activation in acute myeloid leukemia cells. Leukemia 19: 1350-1354, 2005.

18. Shishodia S, Sethi G, Konopleva M, Andreeff $M$ and Aggarwal BB: A synthetic triterpenoid, CDDO-Me, inhibits IkappaBalpha kinase and enhances apoptosis induced by TNF and chemotherapeutic agents through down-regulation of expression of nuclear factor kappaB-regulated gene products in human leukemic cells. Clin Cancer Res 12: 1828-1838, 2006.
19. Chintharlapalli S, Papineni S, Konopleva M, Andreef M, Samudio I and Safe S: 2-Cyano-3,12-dioxoolean-1,9-dien-28-oic acid and related compounds inhibit growth of colon cancer cells through peroxisome proliferator-activated receptor gammadependent and -independent pathways. Mol Pharmacol 68 119-128, 2005.

20. Liby K, Hock T, Yore MM, Suh N, Place AE, Risingsong R, Williams CR, Royce DB, Honda T, Honda Y, et al: The synthetic triterpenoids, CDDO and CDDO-imidazolide, are potent inducers of heme oxygenase-1 and Nrf2/ARE signaling. Cancer Res 65: 4789-4798, 2005

21. Deeb D, Gao X, Jiang H, Dulchavsky SA and Gautam SC: Oleanane triterpenoid CDDO-Me inhibits growth and induces apoptosis in prostate cancer cells by independently targeting prosurvival Akt and mTOR. Prostate 69: 851-860, 2009.

22. Chin YW, Balunas MJ, Chai HB and Kinghorn AD: Drug discovery from natural sources. AAPS J 8: E239-E253, 2006.

23. Itokawa H, Morris-Natschke SL, Akiyama $\mathrm{T}$ and Lee KH: Plantderived natural product research aimed at new drug discovery. $\mathrm{J}$ Nat Med 62: 263-280, 2008.

24. Gao X, Deeb D, Liu Y, Arbab AS, Divine GW, Dulchavsky SA and Gautam SC: Prevention of prostate cancer with oleanane synthetic triterpenoid CDDO-Me in the TRAMP mouse model of prostate cancer. Cancers (Basel) 3: 3353-3369, 2011

25. Deeb D, Gao X, Liu Y, Jiang D, Divine GW, Arbab AS Dulchavsky SA and Gautam SC: Synthetic triterpenoid CDDO prevents the progression and metastasis of prostate cancer in TRAMP mice by inhibiting survival signaling. Carcinogenesis 32: 757-764, 2011.

26. Ling X, Konopleva M,Zeng Z, Ruvolo V, Stephens LC, Schober W McQueen T, Dietrich M, Madden TL and Andreeff M: The novel triterpenoid C-28 methyl ester of 2-cyano-3, 12-dioxoolen-1, 9-dien-28-oic acid inhibits metastatic murine breast tumor growth through inactivation of STAT3 signaling. Cancer Res 67: 4210-4218, 2007

27. Liby K, Royce DB, Williams CR, Risingsong R, Yore MM, Honda T, Gribble GW, Dmitrovsky E, Sporn TA and Sporn MB: The synthetic triterpenoids CDDO-methyl ester and CDDOethyl amide prevent lung cancer induced by vinyl carbamate in A/J mice. Cancer Res 67: 2414-2419, 2007.

28. Datta SR, Dudek H, Tao X, Masters S, Fu H, Gotoh Y and Greenberg ME: Akt phosphorylation of BAD couples survival signals to the cell-intrinsic death machinery. Cell 91: 231-241, 1997.

29. Brunet A, Bonni A, Zigmond MJ, Lin MZ, Juo P, Hu LS, Anderson MJ, Arden KC, Blenis J and Greenberg ME: Akt promotes cell survival by phosphorylating and inhibiting a Forkhead transcription factor. Cell 96: 857-868, 1999.

30. Karin M, Cao Y, Greten FR and Li ZW: NF-kappaB in cancer: From innocent bystander to major culprit. Nat Rev Cancer 2: 301-310, 2002

31. Hay N and Sonenberg N: Upstream and downstream of mTOR. Genes Dev 18: 1926-1945, 2004. 